TITLE:

\title{
Dynamics starting from zero velocities in the classical Coulomb three-body problem
}

\author{
$\operatorname{AUTHOR}(\mathrm{S})$ :
}

Sano, Mitsusada M.

\section{CITATION:}

Sano, Mitsusada M.. Dynamics starting from zero velocities in the classical Coulomb three-body problem. Physical Review E 2007, 75(2(Part 2)): 026203.

ISSUE DATE:

2007-02

URL:

http://hdl.handle.net/2433/59079

RIGHT:

Copyright 2007 American Physical Society 
PHYSICAL REVIEW E 75, 026203 (2007)

\title{
Dynamics starting from zero velocities in the classical Coulomb three-body problem
}

\author{
Mitsusada M. Sano \\ Graduate School of Human and Environmental Studies, Kyoto University, Sakyo, Kyoto, 606-8501, Japan \\ (Received 28 March 2006; revised manuscript received 14 November 2006; published 2 February 2007)

\begin{abstract}
A qualitative geometrical analysis of the classical Coulomb three-body problem is developed. Three ions and atom are investigated: namely, $\mathrm{H}^{-}, \mathrm{He}$, and $\mathrm{Li}^{+}$. The dynamics of these atom and ions is treated in the approximation that the nucleus has infinite mass. Geometrical structures of the dynamics starting from the initial conditions with zero velocities are elucidated. In particular, the distribution of the final states in the initial condition space, its fractal structure, and binary collision orbits are specified. As a result, it is found that binary collision orbits form a lobelike structure. It is shown that the lobelike structure is, in fact, fractal. The lobelike structure is continuously connected to the collinear $e$ Ze configuration space, in which a well-known binary symbolic dynamics exists. With these observations, it is expected that as well as triple-collision orbits, binary-collision orbits also serve us some clue to find a symbolic dynamics for the full dynamics with zero angular momentum.
\end{abstract}

DOI: 10.1103/PhysRevE.75.026203

PACS number(s): 05.45.Mt, 45.50.Jf, 34.10.+x

\section{INTRODUCTION}

The three-body problem in celestial mechanics is one of the oldest problems in physics. Many physicists avoid this problem because of its difficulty, since Poincare showed that general solutions of the three-body problem are not analytically solved. But investigation of the three-body problem is still going on, with the aid of numerical simulation and some mathematical developments [1-3].

Turning our attention to the atomic world, the three-body problem also exists in atom and ions-i.e., systems of two electrons and one nucleus such as $\mathrm{H}^{-}, \mathrm{He}$, and $\mathrm{Li}^{+}$. In particular, the helium atom is the most interesting system. It is a nontrivial actual system of three-body problems. The interest of atomic physicists is oriented to the electron-electron correlation in the helium atom. How do two electrons repulsively interact with each other? At the same time, how are they bound by the nucleus? These two questions are somewhat contradictory. Two electrons are moving, more or less, chaotically in the classical sense. Thus, we ask, how is the correlation of electrons encoded in a chaotic classical dynamics? This is the most mysterious aspect of the helium atom from a classical point of view. Its quantum mechanical behavior is also interesting. Usually our common knowledge on quantum chaos tells us that the spectral statistics of a classically chaotic quantum system obeys the prediction of random matrix theory: namely, Gaussian orthogonal ensemble (GOE), Gaussian unitary ensemble (GUE), and Gaussian symplectic ensemble (GSE) depending on the symmetry which a given system possesses. This is the wellknown Bohigas-Giannoni-Schmit (BGS) conjecture [4]. This conjecture has been recently solved by semiclassical theory [5-7]. According to the BGS conjecture, many readers may think that since the dynamics in the collinear eZe configuration is hard chaos $[13,18]$, the spectrum of the helium atom might obey the spectral statistics of the random matrices. Indeed the full dynamics of the helium atom is a mixture of regular and hard chaotic motions. However, the actual spectrum of the helium atom displays a nice regularity which is characterized by quantum defects or by group-theoretical consideration $[8,9]$. This apparent contradiction with our common sense on quantum chaos is the most interesting point in Coulomb three-body problems such as the helium atom.

In the 1990s, the classical dynamics of $\mathrm{He}$ and $\mathrm{H}^{-}$was investigated mainly for the collinear $e Z e$ configuration and the Gutzwiller trace formula was applied to these systems [10-14]. It is justified that the semiclassical quantization by the Gutzwiller trace formula was successful $[11,12,14]$. Semiclassical eigenenergies coincide with quantum exact ones very well. In particular, some of the quantum defects of helium are explained by intermittency in its classical dynamics [14]. The regularity in the spectrum is originated in the hidden regularity of classical chaotic dynamics. Several results on the helium atom are reviewed in [9].

In this paper, the classical Coulomb three-body problem of $\mathrm{H}^{-}, \mathrm{He}$, and $\mathrm{Li}^{+}$is investigated. Recently, using McGehee's blowup technique [15], the classical Coulomb problem has been investigated for the $e Z e$ collinear configuration with infinite nucleus mass [17], for the $e Z e$ collinear configuration with finite mass [18], and for the two-dimensional case with zero total angular momentum (infinite nucleus mass) [19-22]. This paper's aim is to extend the results of [19-22] to elucidate the dynamical structure in two-electron atoms and ions for the general two-dimensional case with zero angular momentum. The Hamiltonian which describes the systems is given by

$$
H=\frac{\mathbf{p}_{1}^{2}}{2}+\frac{\mathbf{p}_{2}^{2}}{2}-\frac{1}{r_{1}}-\frac{1}{r_{2}}+\frac{1}{Z r_{12}}=E,
$$

where $r_{1}$ (or $r_{2}$ ) is the distance between nucleus and electron 1 (or electron 2) and $r_{12}$ is the distance between electrons 1 and 2. The case that the energy is $E<0$ is considered. The dynamics is described by the approximation that the mass of the nucleus is infinite.

The first investigations of classical dynamics were restricted to one-dimensional dynamics: namely, the collinear $e Z e$ configuration [11,12], the collinear eeZ configuration $[12,13]$, or the Wannier configuration [13], because one- 
dimensional dynamics is simple. Although one-dimensional dynamics is simple, its dynamics exhibits various behaviors. The dynamics is fully chaotic for the eZe configuration, regular for the eeZ configuration, and the mixture of chaotic and regular behaviors for the Wannier configuration. Very recently a systematic investigation of the classical dynamics of the helium atom was started by using McGehee's blowup technique [15] for the one-dimensional case [17-19] and for the two-dimensional case [19-22]. Their investigation focuses on the dynamics of near-triple collisions. Triple collisions are measure-zero events and, in fact, essential singular events, but they form a bone structure of its phase space structure [19-22]. The triple-collision manifold which is a manifold in the phase space related to the triple-collision events is a key tool to elucidate the dynamics near triple collisions. The triple-collision manifold is, at the same time, a dividing surface $(E=0)$ between $E<0$ and $E>0$ in the phase space, where $E$ is the total energy of the system. In this way, the triple-collision manifold determines not only the local structure near triple collisions, but also the global structure of the phase space. Therefore, the triple-collision manifold is a good starting point to investigate the dynamics of the Coulomb three-body problem.

A special case of the above systems-namely, systems with zero total angular momentum-is considered. If the system has zero total angular momentum, the system is confined to a two-dimensional plane and there are possibilities that triple collisions occur. In this case, a sophisticated analytical technique developed by McGehee [15] is very helpful for investigating the phase-space structure. Although McGehee's technique is useful, elucidating the geometrical structure of the whole phase space is not possible at present. In the above setting, the degree of freedom is 3. Since there is energy conservation, the dimension of the whole phase space is 5. In order to simplify the analysis of the phase space, we restrict ourselves to investigate a subspace of the whole phase space, which we will be able to treat. Thus, in this paper, the dynamics starting from zero velocities is investigated for simplicity. It is that three particles with zero velocities (thus, zero total angular momentum) are freely time evolved by their Coulomb interaction. The dimension of the initial condition space is only 2 . This setting enables us to investigate fine structures in the phase space. In the gravitational threebody problem, the dynamics starting from zero velocities is called the free-fall problem. In order to avoid confusion between gravitational and Coulomb systems, the short name "DZV" is used for Coulomb systems. For brevity, DZV stands for dynamics starting from zero velocities.

In the DZV, this initial condition space is just enclosed by the triple-collision manifold and, at the same time, most far from the triple-collision manifold. The distribution of the final states and the position of the binary-collision orbits in the initial condition space are specified. This attempt makes us elucidate the dynamics in some detail. Several observations of characteristic features, including the fractal property of the distribution of the final states and the binary-collision orbits, are found. Among them, it is emphasized that in order to find a symbolic dynamics which describes the dynamics with zero angular momentum, the collinear $e Z e$ dynamics may be a key clue. In fact, it is found that at least in the DZV, the collinear eZe dynamics smoothly continues into a full two-dimensional dynamics with zero angular momentum. Thus near the $e Z e$ configuration, the binary-collision orbits surely give some tool to describe the dynamics in a symbolic way. This observation is found by specifying the position of the binary-collision orbits.

This paper is organized as follows. In Sec. II, the triplecollision manifold (TCM) and the total flow are derived. Although the total flow is not fully regularized, in order to elucidate the phase-space structure, the derived equations of motion and the TCM are needed and useful. In Sec. III, the $\mathrm{DZV}$ is investigated. In particular, in the initial condition space, the set of the initial conditions of triple encounters is specified. In addition, the difference or similarity of dynamical behavior among $\mathrm{H}^{-}, \mathrm{He}$, and $\mathrm{Li}^{+}$is elucidated. In Sec. IV, the method of Tanikawa et al. to find binary collisions, which was originally used for the celestial free-fall problem, is introduced. In Sec. V, numerical results of the method introduced in the previous section are shown. The geometrical structures of the DZV of He are elucidated. Several important observations are summarized. In Sec. VI, a summary and conclusions are given.

\section{TRIPLE-COLLISION MANIFOLD}

The details of the derivation of the triple-collision manifold have been already reported in [19]. Here we sketch its derivation. With zero total angular momentum, the Hamiltonian in the hyperspherical coordinates is given by

$$
\begin{aligned}
H= & \frac{1}{2}\left(p_{r}^{2}+\frac{4 p_{\chi}^{2}}{r^{2}}+\frac{4 p_{\alpha}^{2}}{r^{2} \sin ^{2}(\chi)}\right)-\frac{1}{r}\left(\frac{1}{\cos \left(\frac{\chi}{2}\right)}+\frac{1}{\sin \left(\frac{\chi}{2}\right)}\right. \\
& \left.-\frac{1}{Z[1-\sin (\chi) \cos (\alpha)]^{1 / 2}}\right) \\
& \equiv T+\frac{1}{r} V,
\end{aligned}
$$

where $r_{1}=\cos (\chi / 2), \quad r_{2}=\sin (\chi / 2)$, and the hyperradius $r=\left(r_{1}^{2}+r_{2}^{2}\right)^{1 / 2}$ with $\chi=2 \arctan \left(r_{2} / r_{1}\right)$. The Hamiltonian equations of motion are directly derived. After that, we employ the following transformations: (i) the scaling transformation

$$
u=r^{1 / 2} p_{r}, \quad x=\sin (\chi) r^{-1 / 2} p_{\chi}, \quad w=r^{-1 / 2} p_{\alpha},
$$

(ii) McGehee's blowup transformation

$$
d t^{\prime}=r^{-3 / 2} d t,
$$

and (iii) the time transformation

$$
\frac{d t^{\prime}}{d \tau}=\sin (\chi) .
$$

Finally we have

$$
\frac{d r}{d \tau}=\sin (\chi) r u
$$




$$
\begin{gathered}
\frac{d \chi}{d \tau}=4 x, \\
\frac{d \alpha}{d \tau}=\frac{4 w}{\sin (\chi)}, \\
\frac{d u}{d \tau}=-\sin (\chi)\left(\frac{1}{2} u^{2}+V(\chi, \alpha)-2 r E\right), \\
\frac{d x}{d \tau}=-\sin (\chi) \cos (\chi)\left[u^{2}+2 V(\chi, \alpha)-2 r E\right]-\frac{1}{2} \sin (\chi) u x \\
-\sin 2(\chi) \frac{\partial}{\partial \chi} V(\chi, \alpha), \\
\frac{d w}{d \tau}=-\frac{1}{2} \sin (\chi) u w-\sin (\chi) \frac{\partial}{\partial \alpha} V(\chi, \alpha) .
\end{gathered}
$$

The energy relation is given by

$$
\sin ^{2}(\chi) u^{2}+4 x^{2}+4 w^{2}+2 \sin ^{2}(\chi) V(\chi, \alpha)-2 \sin ^{2}(\chi) r E=0 .
$$

Thus the phase space is defined by

$$
\begin{aligned}
\mathcal{M}= & \left\{(r, \chi, \alpha, u, x, w) \mid \sin ^{2}(\chi) u^{2}+4 x^{2}+4 w^{2}\right. \\
& +2 \sin ^{2}(\chi) V(\chi, \alpha)-2 \sin ^{2}(\chi) r E=0, \\
& r>0,0 \leqslant \chi \leqslant \pi, 0 \leqslant \alpha \leqslant 2 \pi, \\
& -\infty<u, x, w<+\infty\} .
\end{aligned}
$$

The dimension of the whole phase space is $\operatorname{dim}(\mathcal{M})=5$. The flow $\Phi^{\tau}$ is formally defined by Eqs. (6)-(11)_for example, for $\mathbf{X}(0) \in \mathcal{M}$,

$$
\mathbf{X}(\tau)=\Phi^{\tau} \mathbf{X}(0)
$$

The TCM is defined by the energy relation with $r=0$ and/or $E=0$ :

$$
\sin ^{2}(\chi) u^{2}+4 x^{2}+4 w^{2}+2 \sin ^{2}(\chi) V(\chi, \alpha)=0 .
$$

The TCM is

$$
\begin{aligned}
\mathcal{M}_{T C M}= & \left\{(\chi, \alpha, u, x, w) \mid \sin ^{2}(\chi) u^{2}+4 x^{2}+4 w^{2}\right. \\
& +2 \sin ^{2}(\chi) V(\chi, \alpha)=0, \quad 0 \leqslant \chi \leqslant \pi, \quad 0 \leqslant \alpha \leqslant 2 \pi \\
& -\infty<u, x, w<+\infty\} .
\end{aligned}
$$

The final result of the equations of motion is not fully regularized. See Eq. (8). The right-hand sides of these equations diverge at $\chi=0$ and $\pi$. In Ref. [19], it is written that they are regularized. It was a mistake. However, the derived equations of motion are still useful and the stability analysis of the fixed point is reliable.

As shown in $[19,21]$, for the eZe collinear configuration (i.e., $\alpha=\pi$ ), the TCM is topologically equivalent to a spherical shell with four holes, and for the Wannier configuration (i.e., $\chi=\frac{\pi}{2}$ ), the TCM is topologically equivalent to a spherical shell.

The stability analysis of the fixed points of the total flow shows that there exists two fixed points

$$
(r, \chi, \alpha, u, x, w)=\left(0, \frac{\pi}{2}, \pi, u^{( \pm)}, 0,0\right),
$$

where $u^{( \pm)}= \pm \sqrt{-2 V\left(\frac{\pi}{2}, \pi\right)}$. These two fixed points are just on the TCM. We call the plus (or minus) signed fixed point the point $A$ (or $B$ ). We denote the stable and unstable manifolds of points $A$ and $B$ by $\mathcal{W}^{(s)}(A), \mathcal{W}^{(u)}(A), \mathcal{W}^{(s)}(B)$, and $\mathcal{W}^{(u)}(B)$. By the above stability analysis, their dimensions are $\operatorname{dim}\left[\mathcal{W}^{(s)}(A)\right]=3, \operatorname{dim}\left[\mathcal{W}^{(u)}(A)\right]=2, \quad \operatorname{dim}\left[\mathcal{W}^{(s)}(B)\right]=2, \quad$ and $\operatorname{dim}\left[\mathcal{W}^{(u)}(B)\right]=3$.

We use the term "orbit" for $\cup_{\tau=\tau_{\min }}^{\tau_{\max }} \mathbf{X}(\tau)$ in $\mathcal{M}$ or for that in the phase space of the Cartesian coordinates and use the term "trajectory" for the projection of the orbit to the space of the position coordinates.

Finally an important fact will be explained as follows: Where are triple-collision orbits in the phase space? What is the relation between the triple-collision orbits and the TCM? By the definition, the triple-collision orbits behave like

$$
\mathbf{X}(\tau) \in \mathcal{M} \rightarrow \text { some point on } \mathcal{M}_{T C M} \text { as } \tau \rightarrow \infty
$$

and

$$
\mathbf{X}(\tau) \in \mathcal{M} \rightarrow \text { some point on } \mathcal{M}_{T C M} \text { as } \tau \rightarrow-\infty \text {. }
$$

It is clear that the triple-collision orbits are related to the stable and unstable manifolds of the fixed points on the TCM, since the fixed points $A$ and $B$ are on the TCM. Thus some of the triple-collision orbits, which experience a triple collision in the future, are just on $\mathcal{W}^{(s)}(A)$. Note that all of the triple-collision orbits, which will experience a triple collision in the future, are not just on $\mathcal{W}^{(s)}(A)$. As a timereversed relation, some of the triple-collision orbits, which experienced a triple collision in the past, are just on $\mathcal{W}^{(u)}(B) . \mathcal{W}^{(s)}(A)$ and $\mathcal{W}^{(u)}(B)$ probably give information on a partition of the phase space. Therefore the triple-collision orbits are primary important to find a symbolic dynamics for this system. In addition, the binary-collision orbits have secondary importance. The binary collisions have two typesi.e., electron-1 binary collision with nucleus and electron-2 binary collision with nucleus. If both types of binary collisions occur at the same time, it is a triple collision. This fact should be kept in mind up to the last section.

\section{DYNAMICS STARTING FROM ZERO VELOCITIES}

The DZV is that three particles with initial zero velocities are freely time evolved by their interaction. In celestial mechanics, the free-fall problem (i.e., the DZV) has been investigated by Agekian and Anosova [25], by Tanikawa, Umehara, and Abe [26], and by Tanikawa [27]. For the Coulomb three-body problem, there is only a preliminary result in [19]. In this section, the DZV is investigated for $\mathrm{H}^{-}, \mathrm{He}$, and $\mathrm{Li}^{+}$in detail. The TCM is a key tool to understand the phasespace structure of the Coulomb three-body problem, as well as for the gravitational three-body problem, in particular near triple collisions.

The initial condition is as follows. The configuration space is the $(x, y)$ plane. Now three particles have zero velocities. The nucleus is set at the origin. A triangle formed by 


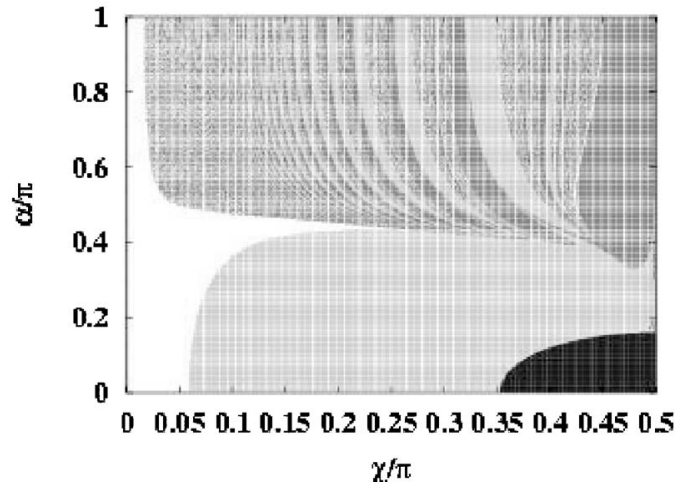

FIG. 1. Initial condition space of the DZV in $D_{1 / 4}: \mathrm{H}^{-}$. Dark gray represents the electron-1 escape. Bright gray represents the electron-2 escape. Black represents the forbidden region $(E>0)$. White represents the trapped trajectory.

three particles is characterized by the coordinates $(\chi, \alpha)$ introduced in the previous section. Electron 1 is set on the positive $x$ axis. Electron 2 is set by the angle $\alpha$. The size of the triangle is determined by the energy relation. For simplicity, the initial value of the angle $\alpha$ is the same as the polar angle from the $x$ axis. In the original DZV, the distance between nucleus and electron 1 is fixed to 1 for simplicity. Thanks to the scaling property of the Coulomb system, one can freely scale the position and momentum coordinates. The initial condition space in this representation will be shown later. In this representation, the initial condition space is the inside of the semicircle with radius 1 in the upper half plane.

The initial condition space for $\chi$ and $\alpha$ is $D=\{(\chi, \alpha): 0$ $\leqslant \chi \leqslant \pi, 0 \leqslant \alpha \leqslant 2 \pi\}$. More precisely, for the total flow, the initial condition space is

$$
\begin{aligned}
\mathcal{M}^{(\text {init })} & =\{(r, \chi, \alpha, u, x, w) \mid u=x=w=0, \quad V(\chi, \alpha)-r E=0, \quad r \\
& >0, \quad 0 \leqslant \chi \leqslant \pi, \quad 0 \leqslant \alpha \leqslant 2 \pi\} .
\end{aligned}
$$

From a geometrical symmetry, it is sufficient to consider a quarter of $D$ denoted by $D_{1 / 4}=\{(\chi, \alpha): 0 \leqslant \chi \leqslant \pi / 2$, $0 \leqslant \alpha \leqslant \pi\}$.

For numerical computation, a set of equations of motion derived in [21] is used, which consists of KustaanheimoStiefel (KS) transformation [28,29] and McGehee's blowup technique [15]. A program which adopts the algorithm in $[23,24]$ is also used for the comparison with the numerical result of the algorithm in [21].

Setting an initial condition-i.e., $\mathbf{X}(0) \in \mathcal{M}^{(\text {init })}$ —and starting dynamics with zero velocities at the time $t=0$-i.e., $\mathbf{X}(\tau)=\Phi^{\tau} \mathbf{X}(0)$ - two electrons move regularly or chaotically. Now we characterize each orbit with an initial condition in $D_{1 / 4}$. See Figs. 1-3. There is a forbidden region in $D$ where the system has a positive energy. Such a region is shown in black and denoted by $D_{b}$ in $D$. Most trajectories end at the escaping of one electron. If electron 1 (or 2) escapes, a dark gray (or bright gray) dot is put in $D . D_{d g}$ (or $D_{b g}$ ) is the dark gray (or bright gray) region in $D$; namely, electron 1 (or 2) escapes, respectively. The escaping condition used is that if the variable $u$ exceeds $u^{(+)}$with $\chi<\pi / 2(\chi>\pi / 2)$, then electron 1 (electron 2) escapes, respectively. Because of the

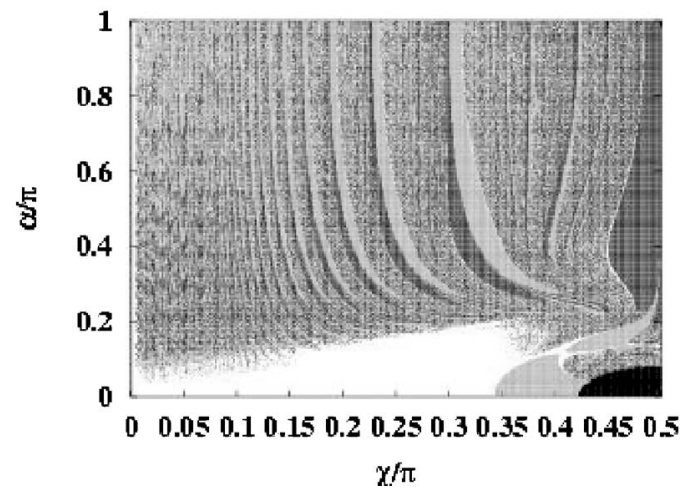

FIG. 2. Initial condition space of the DZV in $D_{1 / 4}$ : He. Dark gray represents the electron-1 escape. Bright gray represents the electron-2 escape. Black represents the forbidden region $(E>0)$. White represents the trapped trajectory.

finiteness of numerical computation-namely, finite timeone cannot follow the whole time evolution. Thus the maximum time of computation is set. It is $\tilde{\tau}_{\max }=50$ in the time variable of Ref. [21] which adopts the parabolic coordinates, KS transform, and McGehee's technique. There is another type of trajectories, where two electrons are still tightly bounded by the nucleus up to the time $\widetilde{\tau}_{\max }$. For such an initial condition, a white dot is plotted in $D$. The white region in $D$ is denoted by $D_{w}$. The trajectories with the initial conditions in $D_{w}$ are either the trajectories which one electron escapes or the trajectories which two electrons are bounded forever. In the latter case, such initial conditions are candidates of tori or more loosely speaking (quasi)periodic orbits.

Here we define some sets. For the DZV, we denote a set of orbits which start and end at triple collisions by $\mathcal{S}_{T C O}^{(0, D)}$. $D$ means the initial condition space. In addition, we denote the set of points, where the orbits in $\mathcal{S}_{T C O}^{(0, D)}$ pass, by $C_{T C O}^{(0, D)}$.

Remark. If an orbit of two electrons is denoted in Cartesian coordinates by the position coordinates

$$
\mathbf{q}(t)=\left(q_{1, x}(t), q_{1, y}(t), q_{2, x}(t), q_{2, y}(t)\right)
$$

and the momentum coordinates

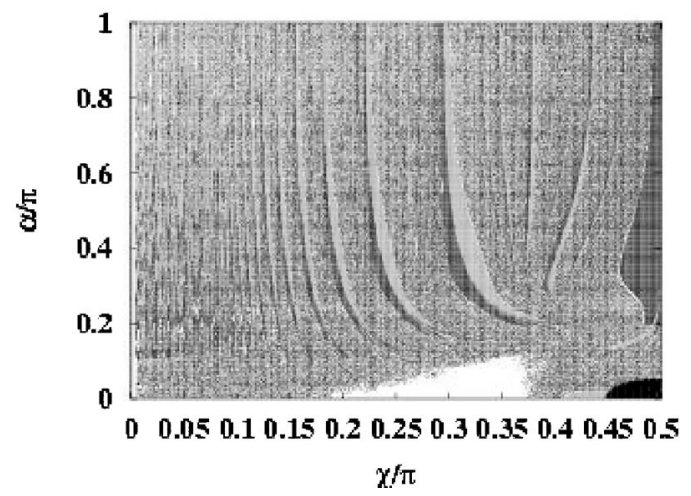

FIG. 3. Initial condition space of the DZV in $D_{1 / 4}: \mathrm{Li}^{+}$. Dark gray represents the electron-1 escape. Bright gray represents the electron-2 escape. Black represents the forbidden region $(E>0)$. White represents the trapped trajectory. 


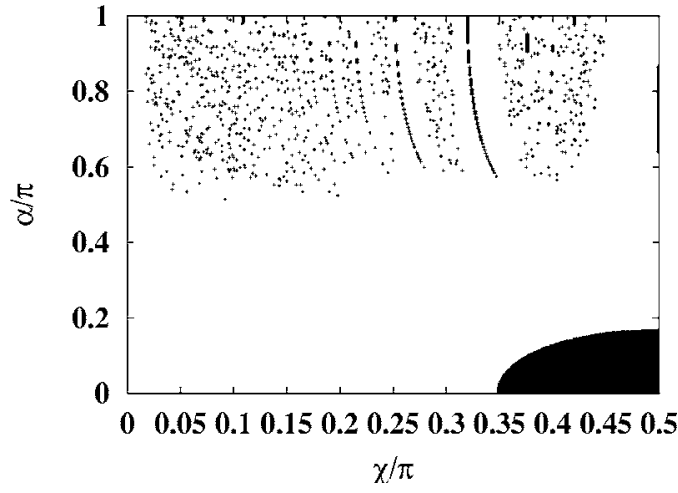

FIG. 4. The first-triple encounter orbits in the initial condition space: $\mathrm{H}^{-}$. Recall that the initial conditions where the triple encounters occur are the boundaries of the bright gray and the dark gray regions in Fig. 1.

$$
\mathbf{p}(t)=\left(p_{1, x}(t), p_{1, y}(t), p_{2, x}(t), p_{2, y}(t)\right),
$$

and at $t=0$, a time evolution with zero velocities starts, then the orbit having the initial condition at $t=0$ in $C_{T C O}^{(0, D)}$ satisfies that

$$
\mathbf{q}(t)=\mathbf{q}(-t), \quad \mathbf{p}(t)=-\mathbf{p}(-t),
$$

where $-t_{*} \leqslant t \leqslant t_{*}$ and $t_{*}$ is the time in which a triple collision occurs in the future. This property is due to a symmetry of the initial condition space $\mathcal{M}^{\text {(init) }}$ and the time-reversed operation. In general, a trajectory starting from a point in $\mathcal{S}_{T C O}^{(0, D)}$ (i.e., in the position coordinates) started from a triple collision at $t=-t_{*}$ and goes to a just turn-back point at $t=0$. Then it will end at a triple collision at $t=t_{*}$. Thus the future trajectory self-retraces the past trajectory in a time-reversed order. This is the most remarkable feature of the trajectory starting from a point in $\mathcal{S}_{T C O}^{(0, D)}$. Note that similarly the periodic orbits in the DZV are self-retracing orbits. That is, at $t=0$, the particles have zero velocities. At half of a period, they also have zero velocities. Then they go back to the initial condition.

Now numerical results are shown. In Figs. 1-3, the results of the DZV for $\mathrm{H}^{-}, \mathrm{He}$, and $\mathrm{Li}^{+}$are presented. There are some differences among them. They shall be explained step by step. First the position where triple encounters occur is checked. In Fig. 4, for $\mathrm{H}^{-}$, the initial conditions that a triple encounter occurs up to the time $\widetilde{\tau}_{\max }=50$ are plotted. Now a criterion for the triple encounter is $0 \leqslant r<0.1$ ( $r$ is the hyperradius). In Fig. 4, there are some curves. It is clear that these curves coincide well with the boundaries between the bright gray region and the dark gray region in $D_{1 / 4}$. This observation suggests that the boundaries $\partial \overline{D_{b g}} \cap \partial \overline{D_{d g}}$ of the bright gray and dark gray regions are related to binary or triple collisions.

Compared with the result of the DZV for all casesnamely, $\mathrm{H}^{-}, \mathrm{He}$, and $\mathrm{Li}^{+}$in Figs. 1-3- the dynamics of $\mathrm{H}^{-}$is a little bit different from that of $\mathrm{He}$ and $\mathrm{Li}^{+}$. This has been already pointed out by Gaspard and Rice [12]. They showed that averaging out the oscillation of fast electron motion and deriving an effective Hamiltonian, the sign of the effective potential changed from $Z=1$ to $Z=2,3, \ldots$. For $Z=1$, there is no stable fixed point (i.e., no frozen planetary orbits). Thus it is thought that this effect is observed in the result of the DZV. Thus Fig. 1 has a large escape region $(0<\alpha<0.5 \pi)$. For $\mathrm{He}$ and $\mathrm{Li}^{+}$, there exist frozen planetary orbits near $\alpha=0$ (i.e., white region).

The most striking feature is that Figs. $1\left(\mathrm{H}^{-}\right), 2(\mathrm{He})$, and $3\left(\mathrm{Li}^{+}\right)$have the stripe structure of the bright gray region and the dark gray region. This stripe structure is nested. This is a typical characteristic feature of chaotic scattering. For $\mathrm{He}$ and $\mathrm{Li}^{+}$, the phase space is roughly separated as the chaotic scattering region and the region of the frozen planetary orbits: namely, chaotic sea and tori.

$\partial \overline{D_{b g}} \cap \partial \overline{D_{d g}}$ forms curves in $D$. This is also a common feature for these three ions and atoms. We searched triplecollision orbits by checking $\partial \overline{D_{b g}} \cap \partial \overline{D_{d g}}$. Our observation implies that the triple-collision orbits are only on the line $\alpha=\pi$. In [26] (gravitational free-fall problem), triplecollision orbits form points in the initial condition space. But there is a difference between them. For the gravitational problem, the Wannier configuration is replaced by the isosceles configuration examined by Devaney [16]. The TCM of the isosceles configuration for the gravitational problem is different from that of the Wannier configuration. The TCM of the isosceles configuration for the gravitational problem is topologically equivalent to a spherical shell with four holes. This difference originates in electron-electron repulsion. Due to the difference of the TCM between both problems, for the gravitational free-fall problem, there exist the triple-collision orbits both for the equilateral type and for the collinear type. On the other hand, for the Coulomb problem, there exist only the triple-collision orbits for the collinear type. The analysis in the later sections supports this observation in a much clearer way.

In addition, there is also similarity among Figs. $2(\mathrm{He})$ and $3\left(\mathrm{Li}^{+}\right)$. They look very similar. This suggests that the underlying dynamics is similar in some sense. However, there is a slight difference among each nested region of $D_{b g}$ and $D_{d g}$ for $\mathrm{H}^{-}, \mathrm{He}$, and $\mathrm{Li}^{+}$. The fractal structure of the nested region gradually changes when $Z$ varies. This implies that, at least, the metrical property is different among the three ions and atoms. If the topological property is different, then the symbolic dynamics for the two-dimensional case with zero total angular momentum is different among them. Therefore, in order to elucidate the topological feature, we should characterize and quantify the stripe structures in the chaotic scattering region carefully. This point will be clarified by checking the binary-collision orbits in the later sections. In addition, the $\alpha=\pi$ is the collinear $e Z e$ configuration. $\partial \overline{D_{b g}} \cap \partial \overline{D_{d g}}$ is continuously connected from the line $\alpha=\pi$ to the region $\alpha \neq \pi$. Therefore, for finding a symbolic dynamics for the two-dimensional case, it is important to examine how the dynamics deformed from $\alpha=\pi$ to $\alpha \neq \pi$. At present, if a symbolic dynamics for the two-dimensional case is found, it is not sure that the number of needed symbols is $5+2=7$ as mentioned in [20]. Here 5 is the number of symbols for the scattering dynamics in two dimensions, and 2 is the number of symbols for the collinear $e Z e$ dynamics. A much more careful investigation is required for acquiring a firm theoretical verification. 
TABLE I. Types of the $n$th minimum: For the types $0,1,2,3$, and 4, we use the gray scale, white, black, gray, dark gray, and none for later figures.

\begin{tabular}{lcccc}
\hline \hline & \multicolumn{2}{c}{$\mathcal{R} \leqslant n$} \\
\cline { 2 - 4 } & Electron-1 collision & Electron-2 collision & $\begin{array}{c}\mathcal{R}<R \text { for } \\
t_{n-1} \leqslant t \leqslant t_{n}\end{array}$ & $\begin{array}{c}\text { Triple collision } \\
\text { before the } n \text {th minimum }\end{array}$ \\
\hline Type 0 & Type 1 & Type 2 & Type 3 & Type 4 \\
$S_{0}$ (white) & $S_{1}$ (black) & $S_{2}$ (gray) & $S_{3}$ (dark gray) & $S_{4}$ (none) \\
\hline \hline
\end{tabular}

\section{SEARCH FOR BINARY COLLISIONS}

Usually the TCM is used for investigating the dynamics near triple collisions. In this study, the opposite aspect is considered-i.e., the phase-space structure far from triple collisions. The initial condition space of the DZV is the most far from the triple-collision manifold. To this direction, we specify the position of binary collisions in the initial condition space and elucidate the fine structure of the DZV.

The free-fall problem in celestial mechanics was, first, investigated by a Russian group-i.e., Agekian and Anosova [25]. There are some results of the free-fall problem [25-27]. Among them, in order to find binary-collision orbits, the algorithm of Tanikawa et al. [26] will be used. Now the precise definition of the binary-collision orbits and the triplecollision orbits are introduced. If the orbit exhibits binary (triple) collisions at some moments, we call such an orbit the binary- (triple-) collision orbit, respectively.

Let us consider an orbit starting with zero velocities. We set

$$
\mathcal{R}(t) \equiv \min \left\{r_{1}(t), r_{2}(t)\right\}
$$

$\mathcal{R}(t)$ is a continuous function with respect to $t$. In general cases, $\mathcal{R}(t)$ has local minima. Let us set $R$ as a fixed distance from the local minimum. $n_{\min }$ stands for the $\left(n_{\min }\right)$ th minimum which we focus on. Let us consider the $n$th minimum for each orbit. There exists four types of orbits. (1) Before an orbit experiences the $n$th minimum, it experiences a triple collision. We call this orbit type 4. (2) $\mathcal{R}(t)$ has $n$ minima and $n-1$ maxima before and including the $n$ minimum. Suppose that at $t=t_{k}$, the $k$ th minimum occurs and at $t=t_{l}^{\prime}$, the $l$ th maximum occurs. If, at $t=t_{n}, \mathcal{R}\left(t=t_{n}\right)>R$, then we call this orbit type 0 . (3) If, for $t_{n-1} \leqslant t \leqslant t_{n}, \mathcal{R}(t) \leqslant R$, then we call this orbit type 3. (4) If, at the $n$th minimum, $\mathcal{R}\left(t_{n}\right)<R$, in this case, we have two possibilities. If $\mathcal{R}\left(t=t_{n}\right)=r_{1}$, then we call this orbit type 1 . If $\mathcal{R}\left(t=t_{n}\right)=r_{2}$, then we call this orbit type 2. All types are listed in Table I. Physically for type-0 trajectories, both electrons 1 and 2 are away from the nucleus. For type-1 (-2) trajectories, electron 1 (2) is close to the nucleus and there is the possibility of a binary collision. For type-3 trajectories, electrons 1 and 2 are not going straight to a nucleus to collide with a nucleus. For type-4 trajectories, the triple collision occurred before the $n$th minimum.

For the $n$th minimum of types 1 and 2, there exists $t_{n}^{*}$ such that $\mathcal{R}\left(t_{n}^{*}\right)=R$ for $t_{n-1}^{\prime} \leqslant t_{n}^{*} \leqslant t_{n}$, We define $\theta$ and $\varphi$ at $t=t_{n}^{*}$ for the $n$th minimum of the type $i(i=1$ or 2$)$

$$
\begin{gathered}
\theta=\arctan \left(y_{i} / x_{i}\right), \\
\varphi=\arctan \left(\dot{y}_{i} / \dot{x}_{i}\right)-\theta-\pi,
\end{gathered}
$$

where $\left(x_{i}, y_{i}\right)$ are the position of the $i$ th electron in the Cartesian coordinates. If $\varphi=0$, electron $i(=1$ or 2$)$ runs into the nucleus in a straight way. This must be a binary collision. In this way, we can specify binary collisions among local minimums of $\mathcal{R}(t)$ by checking the value of $\varphi$.

Lattice points $(2000 \times 2000)$ in $D_{1 / 4}$ are prepared. For each initial condition on each lattice point, we carry out the DZV and check the type of its orbit. See Fig. 5. If the orbit is the type $i(i=0,1,2,3,4)$, the gray scaled dots are plotted at the corresponding lattice point-i.e., 0 (white), 1 (black), 2 (gray), 3 (dark gray), and 4 (none), respectively. Triple collisions are measure-zero events. In this calculation, we cannot specify them. Therefore, for orbits of type 4, we do not give a gray scale. The shaded region is called $S_{i}$ $(i=0,1,2,3,4)$. For numerical simulation, $R$ and $n_{\text {min }}$ are chosen as $R=10^{-1}, 10^{-2}, 10^{-3}$ and $n_{\min }=1,2, \ldots, 8$. In the following section, the results of the above analysis for He are reported.

\section{NUMERICAL RESULTS}

In this section, we report the results of the DZV for He by the analysis based on the previous section.

In Fig. 5, the classification of the types of orbits in $D_{1 / 4}$ for He is depicted. This type of figures up to $n_{\min }=8$ is actually numerically calculated. But because of a shortage of space, it is plotted only for $n_{\text {min }}=1,2,3,4$. Near $\alpha=\pi$, there is a stripe structure of the black and gray regions-i.e., $S_{1}$ and $S_{2}$. The first important observation is as follows.

Observation 1. For the figure of the $\left(n_{\min }\right)$ th minimum, the number of vertical stripes of black and gray regions (i.e., $S_{1}$ and $S_{2}$ ) is just $2^{n_{\text {min }}-1}$ in $D_{1 / 4}$.

In Sec. III, the figures that represent the final states in the initial condition space are shown. The final state means which electron escapes: namely electron 1 or 2 . It is found that there is a stripe structure of the final states. In fact, the stripe structure there is fractal. This observation in Sec. III is very similar to observation 1 . The space of $\alpha=\pi$ is just the collinear $e Z e$ space. Therefore, observation 1 implies that the description by binary symbolic dynamics might, more or less, continue to the region $\alpha \neq \pi$ but near $\alpha=\pi$. In Fig. 5, the white region $\left(S_{3}\right)$ is large. This is dependent on the choice 

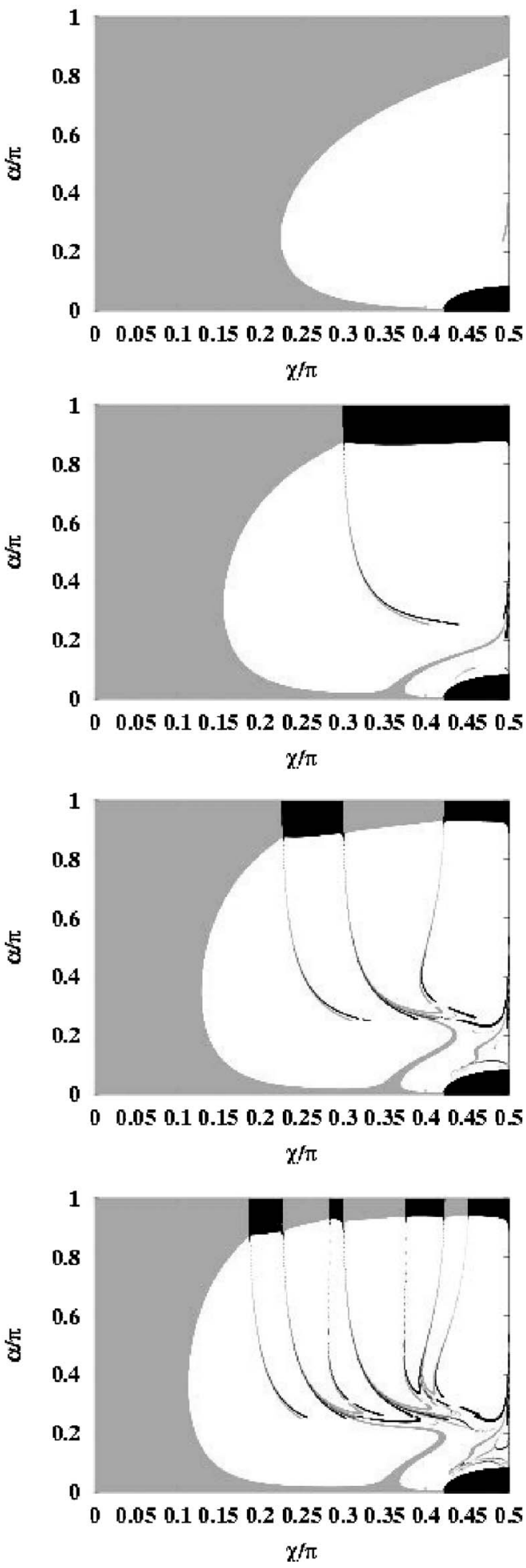

FIG. 5. Classification of the initial condition space $D_{1 / 4}$ for He: (a) $n_{\min }=1$, (b) $n_{\min }=2$, (c) $n_{\min }=3$, and (d) $n_{\min }=4$. We set $R=10^{-3} \cdot S_{0}$ (white), $S_{1}$ (black), $S_{2}$ (gray), $S_{3}$ (dark gray), and $S_{4}$ (none). The black region in lower right corner is a forbidden region $(E \geqslant 0)$.

of value of $R$. If $R$ is small, the white region $\left(S_{3}\right)$ becomes large and the black and gray regions $\left(S_{1}\right.$ and $\left.S_{2}\right)$ become narrow. But we expect that the narrow black and gray regions do not vanish for finite values of $R$.
In Fig. 6, the binary-collision orbits are plotted for $n_{\min }=1,2,3,4,5,6$. The black (bright gray) curve corresponds to the electron-1 (-2) collisions, respectively. In Fig. 6(a), the binary-collision orbits exist near $\chi=\pi / 2$-i.e., near the Wannier configuration. From the starting the DZV, these binary-collision orbits directly hit nucleus at the first encounter with the nucleus. In Figs. 6(b)-6(f), the different types of binary-collision orbits are observed. We found the following characteristic feature.

Observation 2. The binary-collision orbits form curves - in other words, a lobelike structure in $D_{1 / 4}$. It is expected that almost all lobes start and end at $\alpha=\pi$. In addition, the end points at $\alpha=\pi$ seem to coincide with the boundary between the black and gray regions (i.e., $S_{1}$ and $S_{2}$ ) in Fig. 5. More precisely, the binary-collision orbits exist near the boundary between the black and gray regions in Fig. 5 . Thus the number of lobes is closely related to observation 1 ; i.e., the number of the lobes is $2^{n_{m i n}-1}\left(2^{n_{m i n}-2}\right.$ black lobes and $2^{n_{m i n}-2}$ bright gray lobes). The lobelike structure is formed from the large- $\chi$ region to the small- $\chi$ region in $D_{1 / 4}$ increasing $n_{\text {min }}$.

Figures 6(a)-6(f) are a little bit incomplete because of the finiteness of the lattice size. We suppose that near $\alpha=\pi$, the lobelike structure continues to $\alpha=\pi$. It is clear that the lobelike structure is related to observation 1. If the binarycollision orbits are perturbed slightly, then it is not a binarycollision orbit any more. One electron draws near the elliptic curve (i.e., trapped) or near the parabolic curve (i.e., escape). Thus depending on the perturbation, it falls into the black region or the gray region. This implies that around the boundary region of the black and the gray regions, many kinds of orbits accumulate.

Even though the initial condition space is far from the triple-collision manifold, the binary-collision orbits have information on the triple collisions. The following observation can be used for specifying the triple-collision orbits.

Observation 3. The different kinds of binary-collision orbits (black and bright gray curves), at least, cross at $\alpha=\pi$. These points must be triple-collision orbits, because at these points, the corresponding orbits exhibit not only electron-1 collisions but also electron-2 collisions.

Thus, in Fig. 6, the crossing points of the black and bright gray curves are candidates for triple collisions. But except the points on the line $\alpha=\pi$, we cannot find possibilities of triple-collision points. A more detailed analysis is needed for triple-collision orbits in $D_{1 / 4}$.

The lobelike structure has an interesting feature. If we superpose lobelike structures for $n_{\min }=1,2, \ldots$ into one figure (Fig. 7), the following thing is found.

Observation 4. The lobelike structure is fractal.

In Fig. 8, a schematic picture of the lobelike structure is depicted. As shown in Fig. 8, the lobelike structure for $n_{\min }=n+1$ is nested in the lobelike structure for $n_{\min }=n$. This process must be endlessly continued. Thus the whole lobelike structure is fractal. Each lobe can be numbered by using binary symbols. The curve of the binary-collision orbits is the dividing curve between electron-1 escape and electron-2 escape. Thus this must become an explanation of the stripe structure of the final-state distribution as shown in Sec. III. 

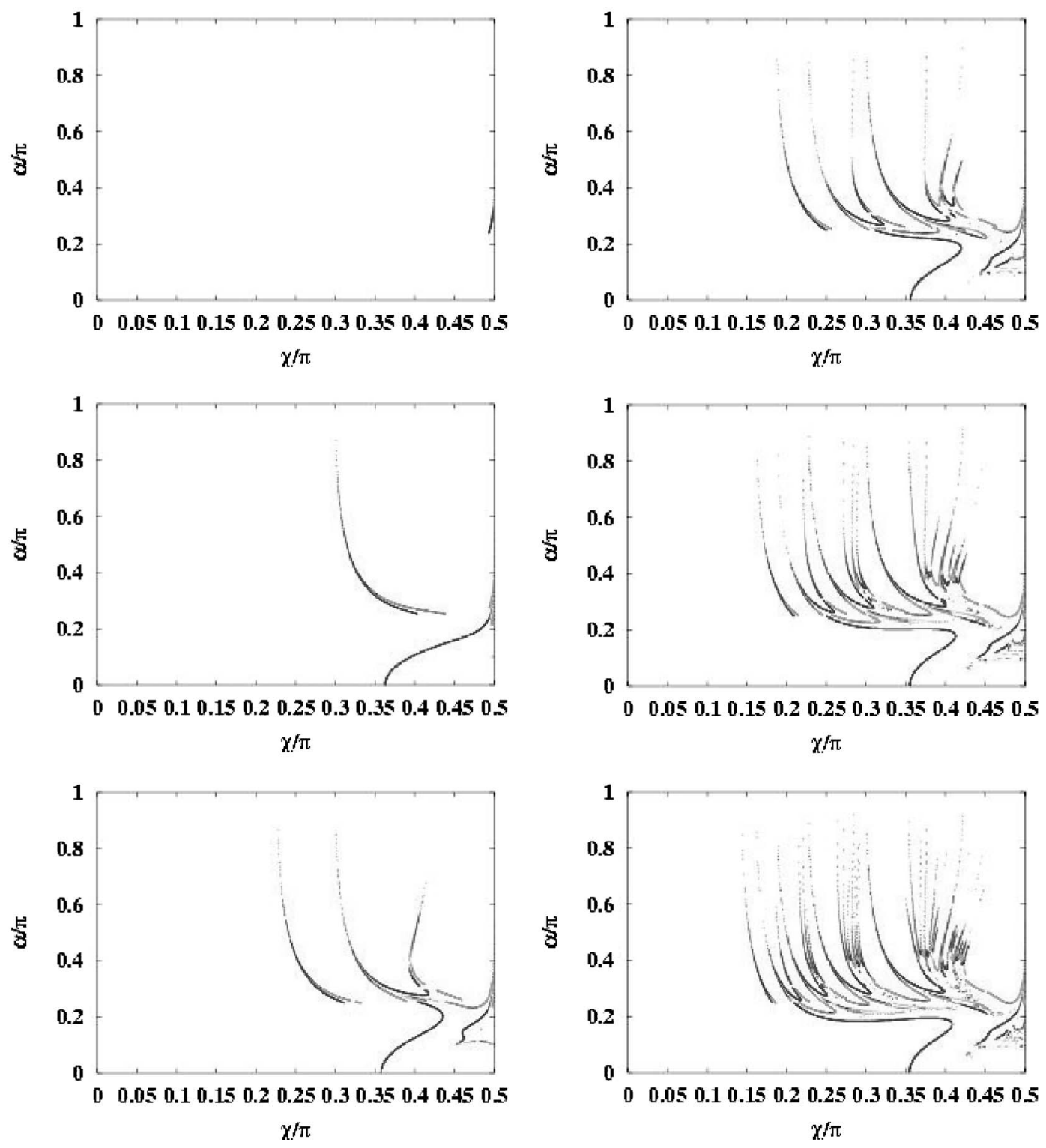

FIG. 6. Binary collision orbits for He: (a) $n_{\min }=1$, (b) $n_{\min }=2$, (c) $n_{\min }=3$, (d) $n_{\min }=4$, (e) $n_{\min }=5$, and (f) $n_{\min }=6$. We set $R=10^{-3}$. The black (bright gray) curve corresponds to the electron-1 (-2) collisions, respectively.

\section{SUMMARY AND CONCLUSIONS}

In this paper, we have reported the results of the DZV for $\mathrm{H}^{-}, \mathrm{He}$, and $\mathrm{Li}^{+}$, especially $\mathrm{He}$, by investigating how the binary-collision orbits are distributed in the initial condition space. First, we examined the distribution of the final states in the initial condition space. For each atom, it has the stripe structure which forms a fractal structure. For further firm characterization of the observed geometrical structure, the position of the binary-collision orbits is specified. It has been shown that the binary-collision orbits form curves in the initial condition space. These curves have a characteristic geometrical structure: namely, the lobelike structure. By a detailed analysis, it is shown that the lobelike structure is, in fact, fractal. The route of the lobelike structure is in the collinear $e Z e$ space-i.e., $\alpha=\pi$. Thus the the lobelike structure might be closely related to the Markov partition in the collinear $e Z e$ space. Thereby the lobelike structure is character-

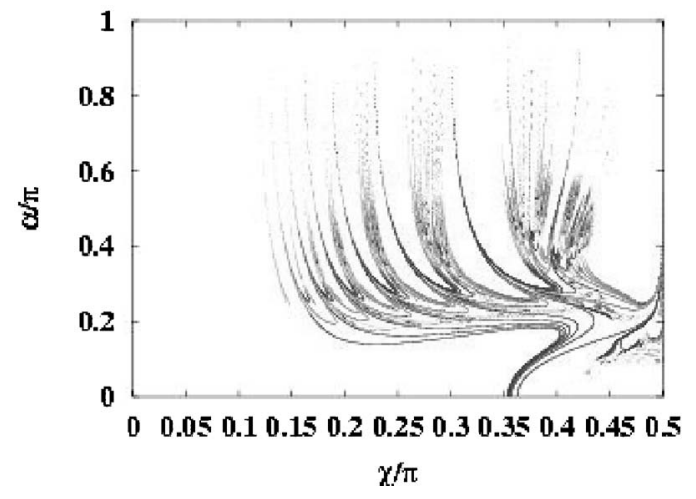

FIG. 7. Binary collision orbits for He: the binary collision orbits from $n_{\min }=1$ to $n_{\min }=8$ are superposed in one figure. $R=10^{-3}$. The black (bright gray) curve corresponds to the electron-1 (-2) collisions, respectively. 


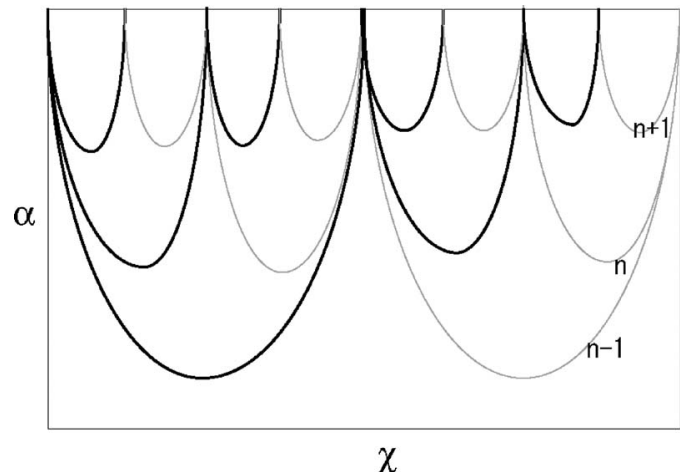

FIG. 8. A schematic picture of the lobelike structure: The $(n+1)$ th lobes are nested in the $n$th lobes. The black (bright gray) curve corresponds to the electron-1 (-2) collisions, respectively.

ized by binary symbols and has $2^{n_{\min }-1}$ lobes for the case of the $\left(n_{\min }\right)$ th minimum. This is a central result of this paper.

A recent investigation has shown dynamics near triple collisions and dynamics with $E=0$ [20-22]. It has been emphasized there that the $e Z e$ collinear dynamics is important and the Markov partition in this space still survives in the full dynamics with zero angular momentum, in some sense. This is consistent with our observations.

In order to find a partition for a symbolic dynamics of this system, as for the usual horseshoe dynamics, one must elucidate entanglement of the stable and unstable manifolds of the fixed points $A$ and $B$. If it is achieved, one can obtain how the partition is formed more or less. As mentioned in the end of Sec. II, the triple-collision manifold is related to the stable and unstable manifolds of the fixed points $A$ and $B$. Thus, in order to obtain the partition for a symbolic dynam- ics, one should know the geometrical structure of the triplecollision orbits. The triple-collision orbits are singular orbits. They must be boundaries of the partition which we want. In addition, the triple-collision orbits are related to the binarycollision orbits in an indirect way. Therefore, it is thought that in order to find a symbolic dynamics for the full dynamics with zero angular momentum, the geometrical structure of the triple-collision orbits must provide a primary clue and that the binary-collision orbits a seconary clue.

In [30], they investigate the existence of periodic orbits for the celestial free-fall problem. As mentioned in Sec. III, the periodic orbits in the DZV must be self-retracing. This property would be very useful to find this type of periodic orbits. For the DZV for the Coulomb system, the treatment of [30] might work.

In the present status, since our investigation was limited to a subspace of the whole phase space, we do not still know much about the geometrical structure of the phase space of the Coulomb three-body problem with zero angular momentum-i.e., binary-collision orbits, triple-collision orbits, and periodic orbits. Further careful investigation of them should be continued. We believe that such an attempt would lead to finding a symbolic dynamics of the twodimensional Coulomb three-body problem. Then it will be linked to the hidden structure of the energy spectrum of $\mathrm{He}$, $\mathrm{H}^{-}$, and $\mathrm{Li}^{+}$.

\section{ACKNOWLEDGMENTS}

The author thanks Professor K. Tanikawa for useful discussions on the celestial three-body problem. This work was supported by a Grant-in-Aid for Scientific Research (No. 17740252) from the Ministry of Education, Culture, Sports, Science and Technology, Japan.
[1] F. Diacu and P. Holmes, Celestial Encounter: The Origin of Chaos and Stability (Princeton University Press, Princeton, 1996).

[2] M. Valtonen and H. Karttunen, The Three-Body Problem (Cambridge University Press, Cambridge, England, 2006).

[3] C. L. Siegel and J. K. Moser, Lectures on Celestial Mechanics (Springer, Berlin, 1971).

[4] O. Bohigas, M. J. Giannoni, and C. Schmit, Phys. Rev. Lett. 52, 1 (1984).

[5] M. Sieber and K. Richter, Phys. Scr. T90, 128 (2001); M. Sieber, J. Phys. A 35, L613 (2002).

[6] S. Müller, S. Heusler, P. Braun, F. Haake, and A. Altland, Phys. Rev. Lett. 93, 014103 (2005).

[7] S. Müller, S. Heusler, P. Braun, F. Haake, and A. Altland, Phys. Rev. E 72, 046207 (2005).

[8] D. A. Herrick, Adv. Chem. Phys. 52, 1 (1983).

[9] G. Tanner, K. Richter, and J.-M. Rost, Rev. Mod. Phys. 72, 497 (2000).

[10] K. Richter and D. Wintgen, Phys. Rev. Lett. 65, 1965 (1990).

[11] G. S. Ezra, K. Richter, G. Tanner, and D. Wintgen, J. Phys. B 24, L413 (1991).

[12] P. Gaspard and S. A. Rice, Phys. Rev. A 48, 54 (1993).

[13] K. Richter, G. Tanner, and D. Wintgen, Phys. Rev. A 48, 4182 (1993).
[14] G. Tanner and D. Wintgen, Phys. Rev. Lett. 75, 2928 (1995).

[15] R. McGehee, Invent. Math. 27, 191 (1974).

[16] D. L. Devaney, Invent. Math. 60, 249 (1980).

[17] Z.-Q. Bai, Y. Gu, and J. M. Yuan, Physica D 118, 17 (1998).

[18] M. M. Sano, J. Phys. A 37, 803 (2004).

[19] M. M. Sano, Adv. Chem. Phys. 130, Part A, 305 (2005).

[20] N. N. Choi, M.-H. Lee, and G. Tanner, Phys. Rev. Lett. 93, 054302 (2004).

[21] M.-H. Lee, G. Tanner, and N. N. Choi, Phys. Rev. E 71, 056208 (2005).

[22] M.-H. Lee, N. N. Choi, and G. Tanner, Phys. Rev. E 72, 066215 (2005).

[23] S. Mikkola and K. Tanikawa, Celest. Mech. Dyn. Astron. 74, 287 (1999).

[24] S. Mikkola and K. Tanikawa, Mon. Not. R. Astron. Soc. 310, 745 (1999).

[25] T. A. Agekian and J. P. Anosova, Astron. Zh. 44, 1261 (1967).

[26] K. Tanikawa, H. Umehara, and H. Abe, Celest. Mech. Dyn. Astron. 62, 335 (1995).

[27] K. Tanikawa, Celest. Mech. Dyn. Astron. 76, 157 (2000).

[28] P. Kustaanheimo and E. Stiefel, C. R. Hebd. Seances Acad. Sci. 260, 805 (1965).

[29] S. J. Aarseth and K. Zare, Celest. Mech. 10, 185 (1974).

[30] K. Tanikawa, L. Benet, and A. Mylläri (private communication). 